\title{
Uso de substratos alternativos na produção de mudas de pimenta e pimentão
}

Lucely Pereira da Silva, Alessandra Conceição de Oliveira, Naiara Ferreira Alves, Valéria Lima da Silva, Toshik larley da Silva

Universidade Federal de Viçosa - UFV, MG. E-mail: iarley.toshik@gmail.com

\section{Resumo}

A produção de mudas com altos padrões de qualidade requer substratos que disponibilizem características químicas e físicas propícias para tal finalidade. Com isso, objetivou-se avaliar substratos alternativos na produção de mudas de pimentas e pimentões. Comparou-se os seguintes compostos: substrato comercial (Carolina Soil ${ }^{\circ}$ ), areia lavada + casca de arroz carbonizada + esterco bovino $(1: 1: 1 \mathrm{v} / \mathrm{v} / \mathrm{v})$, areia lavada + casca de arroz carbonizada + húmus de minhoca $(1: 1: 1 \mathrm{v} / \mathrm{v} / \mathrm{v})$, Carolina Soil ${ }^{\bullet}+$ casca de arroz carbonizada + esterco bovino (1:1:1 v/v/v) e Carolina Soil + casca de arroz carbonizada + húmus de minhoca (1:1:1 v/v/v). Foram conduzidos dois experimentos simultaneamente, ambos realizados em delineamento de blocos com os tratamentos ao acaso. O primeiro em esquema fatorial cinco (substratos) $x$ dois (cultivares de pimenta), com quatro repetições; e o segundo experimento em esquema fatorial cinco (substratos) x três (cultivares de pimentão), com quatro repetições. Foram avaliadas o índice de velocidade de emergência, porcentagem de emergência, altura de parte aérea, diâmetro de colo, número de folhas e massas frescas e secas da parte aérea, raiz e total. Com base nas características avaliadas, o substrato comercial Carolina Soil ${ }^{\circ}$ foi o mais adequado para a produção de mudas de pimenta e pimentão. Como substrato alternativo, recomenda-se o substrato composto por substrato comercial + casca de arroz + esterco bovino.

Palavras-chave: Capsicum spp.; Capsicum annum; adubação; matéria orgânica.

Use of alternative substrates on production of pepper and sweet pepper seedlings

\begin{abstract}
The seedlings production with high quality standards requires substrates that provide chemical and physical characteristics suitable for this purpose. Therefore, was evaluated alternative substrates in the production of seedlings of peppers and sweet peppers. For this purpose, the following compounds were compared: commercial substrate (Carolina Soil ${ }^{\circledR}$ ), washed sand + carbonized rice straw + bovine manure $(1: 1: 1 \mathrm{v} / \mathrm{v} / \mathrm{v})$, washed sand + carbonized rice straw + earthworm humus $(1: 1: 1 \mathrm{v} / \mathrm{v} / \mathrm{v})$, Carolina Soil ${ }^{\circledR}+$ carbonized rice straw + bovine manure $(1: 1: 1 \mathrm{v} / \mathrm{v} / \mathrm{v})$ and Carolina Soil ${ }^{\circledR}+$ carbonized rice straw + earthworm humus (1:1:1 $\mathrm{v} / \mathrm{v} / \mathrm{v})$. Two experiments were carried out simultaneously, both performed in a randomized block design. The first one was carried out in factorial scheme 5 (substrates) $\times 2$ (pepper cultivars), with four replications. The second experiment was carried out in a factorial scheme 5 (substrates) $\times 3$ (sweet pepper cultivars), with four replications. The emergence speed index, emergency percentage, shoot height, stem diameter, number of leaves, and fresh and dry masses of shoot, root and total were evaluated. Based on the evaluated characteristics, the commercial substrate Carolina Soil ${ }^{\circledR}$ was the most suitable for the production of pepper and sweet pepper seedlings. As an alternative substrate, the substrate composed of commercial substrate + carbonized rice straw + bovine manure is recommended.
\end{abstract}

Keywords: Caspsicum spp.; Caspsicum annum; fertilizing; organic matter. 


\section{Introdução}

Dentre as hortícolas mais expressivas está o gênero Capsicum pertencente à família Solanaceae, que envolve as pimentas (Capsicum spp.) e pimentões (Capsicum annum) (BÜTTOW et al., 2010). A pimenta e o pimentão são originários da América Central e do Sul, atualmente existem cerca 25 espécies catalogadas, sendo que apenas cinco são domesticadas (SILVA et al., 2008).

Nos últimos anos, o consumidor brasileiro vem se preocupando cada vez mais com a qualidade dos alimentos que consome. Para suprir os requisitos e demanda do mercado, o cultivo de hortaliças foi impulsionado, melhorando as práticas de manejo a fim de conservar a qualidade do produto (TRENTO et al., 2011). Nas últimas décadas, a crescente demanda e a exigência por hortaliças de melhor qualidade contribuíram para a inserção de novas tecnologias na cadeia produtiva (SILVA et al., 2008).

O transplantio de mudas é uma opção para minimizar perdas e assegurar benefícios como maior uniformidade da lavoura, eliminação do desbaste, redução do ciclo da cultura, economia de insumos (SAIDELLES et al., 2009). A formação de mudas é uma fase crucial na produção de hortaliças, tendo em vista que influencia diretamente na produção e no desempenho da cultura implantada (MACIEL et al., 2017). Mudas de boa qualidade se desenvolvem melhor e consequentemente, propiciarão boa formação do sistema radicular, com melhor capacidade de adaptação ao novo local após o transplante, afetando positivamente a sua produção. A produção das mudas está condicionada a utilização de substratos que devem apresentar propriedades físicas, biológicas e químicas desejáveis (ARAÚjO et al., 2013).

Os substratos são essenciais na qualidade das mudas, e devem apresentar boas condições de umidade, macroporos e microporosidade, disponibilidade de nutrientes e de água, capacidade de troca de cátions e boa associação às raízes (NADAl et al., 2015; COSTA et al., 2015), além de ser isento de patógenos e ter baixo teor de sais. Atualmente, existem vários substratos comerciais prontos para o uso, mas o seu valor agregado compromete o rendimento do produtor (GONÇALVES et al., 2016). Em busca de alternativas para reduzir custos e manter o rendimento e a qualidade na produção, o produtor tem adotado o uso de substratos alternativos (NADAl et al., 2015).

O substrato alternativo, geralmente, é oriundo de matérias-primas disponíveis próximo aos locais de cultivo, tendo como principal vantagem a redução de insumos químicos, contribuindo consequentemente para o maior equilíbrio ambiental, além de não onerar o custo para a produção do substrato (COSTA et al., 2013). A mistura de diferentes componentes para a composição de um substrato estável é essencial, dentre os recursos alternativos que vem sendo utilizados ultimamente se destaca os resíduos de origem vegetal e animal (GONÇALVES et al., 2016). Dentre os principais resíduos alternativos utilizados, o esterco e o húmus de minhoca são os mais usados para compor substrato, pois disponibilizam nutrientes e apresentam custo reduzido (DINIZ et al., 2006).

O uso de um único material para compor o substrato se torna inviável, pois não atende as necessidades da cultura, para se conseguir um substrato ideal é importante o uso de misturas de materiais orgânicos (ARAÚJO NETO et al., 2009). Com vista nisso, objetivou-se avaliar o uso de combinações de substratos, a fim de identificar os substratos mais adequado para a produção de mudas de pimentas e pimentões.

\section{Material e Métodos}

O experimento foi conduzido na Universidade do Estado de Mato Grosso (UNEMAT), no Campus de Nova Xavantina-MT, sendo localizado nas coordenadas 140 40' 24" SE, $52^{\circ} 21^{\prime} 11^{\prime \prime} \mathrm{W}$, com altitude de $275 \mathrm{~m}$. A região possui clima tropical do tipo Aw de acordo com a classificação de Köppen, com duas estações bem definidas, sendo que o verão chuvoso que vai de outubro a abril e o inverno seco maio a setembro, tendo a pluviosidade média anual de 1498 mm (ALVES et al., 2011).

Foram realizados simultaneamente dois experimentos no viveiro na UNEMAT, Campus de Nova Xavantina no período de 18 de janeiro a 01 março de 2016. O primeiro experimento foi composto pelas cultivares Pimenta Biquinho Amarela (Capsicum chinense) e Pimenta Malagueta (Capsicum frutescens). No segundo experimento foram avaliados três cultivares de Pimentão, sendo eles Pimentão Quadrado Vermelho, Pimentão Amarelo SF 134 e Pimentão Verde Yolo Wonder (Capsicum annuum).

Os experimentos foram realizados em delineamento em blocos com os tratamentos ao 
acaso, em esquema fatorial cinco (substratos) $x$ dois (cultivares de pimenta) totalizando 10 tratamentos para o primeiro experimento, e em esquema fatorial cinco (substratos) $x$ três (cultivares de pimentão) totalizando 15 tratamentos para o segundo experimento, com quatro repetições e 20 sementes por parcela, sendo consideradas para avaliação as 12 plântulas centrais. Os tratamentos do primeiro experimento foram formados da combinação da mistura de diferentes substratos e das diferentes cultivares. As misturas para a formação dos substratos foram formadas a partir de: substrato comercial Carolina Soil ${ }^{\circledR}$ (CS - testemunha), areia lavada (AL), casca de arroz carbonizada (CAC), húmus de minhoca (HM) e esterco bovino (EB). Os tratamentos para o primeiro experimento foram compostos das seguintes combinações: $\mathrm{T} 1$ : CS e cultivar PBA (pimenta biquinho amarela); T2: $A L+C A C+E B(1: 1: 1 \mathrm{v} / \mathrm{v} / \mathrm{v})$ e cultivar PBA; T3: AL + CAC + HM (1:1:1 v/v/v) e cultivar PBA; T4: CS + CAC + EB (1:1:1 v/v/v) e cultivar PBA; T5: CS + CAC + HM (1:1:1 v/v/v) e cultivar PBA; T6: CS e cultivar PM (pimenta malagueta); T7: AL + CAC + EB (1:1:1 v/v/v) e cultivar PM; T8: AL + CAC + HM (1:1:1 v/v/v) e cultivar PM; T9: CS + CAC + EB (1:1:1 v/v/v) e cultivar PM; T10: CS + CAC + HM (1:1:1 $\mathrm{v} / \mathrm{v} / \mathrm{v}$ ) e cultivar PM.

Os tratamentos para o segundo experimento foram compostos das seguintes combinações: T1: T1: CS e cultivar PQV (pimentão quadrado vermelho); T2: AL + CAC + EB (1:1:1 v/v/v) e cultivar PQV; T3: AL + CAC + HM (1:1:1 v/v/v) e cultivar PQV; T4: CS + CAC + EB (1:1:1 v/v/v) e cultivar PQV; T5: CS + CAC + HM (1:1:1 v/v/v) e cultivar PQV; T6: CS e cultivar PASF (Pimentão Amarelo SF 134); T7: AL + CAC + EB (1:1:1 v/v/v) e cultivar PASF; T8: AL + CAC + HM (1:1:1 v/v/v) e cultivar PASF; T9: CS + CAC + EB (1:1:1 v/v/v) e cultivar PASF; T10: CS + CAC + HM (1:1:1 v/v/v) e cultivar PASF; T11: CS e cultivar PVYW (Pimentão Verde Yolo Wonder); T12: AL + CAC + EB (1:1:1 v/v/v) e cultivar PVYW; T13: AL + CAC + HM (1:1:1 v/v/v) e cultivar PVYW; T14: CS + CAC + EB (1:1:1 v/v/v) e cultivar PVYW; T15: CS + CAC + HM (1:1:1 v/v/v) e cultivar PVYW.

A relação das misturas e suas respectivas proporções em volume foram analisadas pelo Laboratório Terra Análises para Agropecuária de Goiânia-GO e foram determinados os teores de macronutrientes ( $\mathrm{N}, \mathrm{P}, \mathrm{K}, \mathrm{Ca}, \mathrm{Mg}$ e $\mathrm{S}$ em \%) e micronutrientes ( $\mathrm{B}, \mathrm{Cu}, \mathrm{Fe}, \mathrm{Mn}$ e $\mathrm{Zn}$ em $\mathrm{mg} \mathrm{kg}^{-1}$ ), pH e condutividade elétrica (CE) (Tabela 1). Não foi realizado análise química do substrato comercial Carolina Soil ${ }^{\bullet}$ pois na embalagem já continha as especificações. O substrato Carolina Soil ${ }^{\circ}$, de acordo com especificações da empresa produtora, apresenta: porosidade total $(\%)=76$; condutividade elétrica $\left(\mathrm{dS} \mathrm{m}^{-1}\right)=1,5 ; \mathrm{pH}=5,65 ; \mathrm{N}$ $=10 ; \mathrm{P}=20 ; \mathrm{K}=24 ; \mathrm{Cu}=0,05 ; \mathrm{Fe}=0,25 ; \mathrm{Mn}=$ 0,$08 ; \mathrm{B}=0,02 ; \mathrm{Mo}=0,01 ; \mathrm{Zn}=0,05$.

Tabela 1. Resultados da análise química para macro e micronutrientes, $\mathrm{pH}$ e condutividade elétrica (CE) dos substratos alternativos utilizados em experimento de produção de mudas de pimenta e pimentão.

\begin{tabular}{|c|c|c|c|c|c|c|c|}
\hline \multirow{2}{*}{ Substrato } & Matéria Orgânica & $\mathbf{N}$ & $\mathbf{P}$ & K & $\mathrm{Ca}$ & Mg & $\mathbf{S}$ \\
\hline & $\%$ & \multicolumn{6}{|c|}{$\%$} \\
\hline SC & 14,9 & 0,4 & 0,16 & 0,48 & 0,43 & 0,19 & 0,04 \\
\hline$A+C A C+E B$ & 8,0 & 0,1 & 0,16 & 0,48 & 0,36 & 0,21 & 0,12 \\
\hline $\mathrm{A}+\mathrm{CAC}+\mathrm{HM}$ & 18,6 & 0,9 & 0,36 & 0,72 & 1,07 & 1,30 & 0,32 \\
\hline \multirow[t]{3}{*}{$\mathrm{SC}+\mathrm{CAC}+\mathrm{EB}$} & 19,9 & 0,8 & 0,28 & 0,64 & 0,60 & 0,61 & 0,20 \\
\hline & CE & $\mathrm{Cu}$ & $\mathrm{Fe}$ & $M n$ & $\mathrm{Zn}$ & \multirow{2}{*}{\multicolumn{2}{|c|}{$\mathrm{pH}$}} \\
\hline & $\mathrm{mS} \mathrm{cm}-1$ & \multicolumn{4}{|c|}{$\mathrm{mg} \mathrm{kg}^{-1}$} & & \\
\hline SC & 0,635 & 13 & 4650 & 176 & 55 & \multicolumn{2}{|c|}{8,2} \\
\hline$A+C A C+E B$ & 0,751 & 19 & 17900 & 211 & 59 & \multicolumn{2}{|c|}{8,1} \\
\hline $\mathrm{A}+\mathrm{CAC}+\mathrm{HM}$ & 0,715 & 31 & 8490 & 380 & 131 & \multicolumn{2}{|c|}{7,6} \\
\hline $\mathrm{SC}+\mathrm{CAC}+\mathrm{EB}$ & 0,936 & 28 & 27410 & 337 & 90 & \multicolumn{2}{|c|}{7,3} \\
\hline
\end{tabular}

SC: Substrato comercial; A+CAC+EB: areia fina lavada + casca de arroz carbonizada + esterco bovino; $A+C A C+H M$ : areia fina lavada + casca de arroz carbonizada + húmus de minhoca; SC+CAC+EB: substrato comercial + casca de arroz carbonizada + esterco bovino; SC+CAC+HM: substrato comercial + casca de arroz carbonizada + húmus de minhoca. 
A semeadura foi realizada em bandejas de poliestireno expandido (Isopor ${ }^{\circledast}$ ) de 200 células, as quais foram previamente preenchidas com os respectivos substratos e em cada célula foram colocadas três sementes, as fileiras de células vazias foram utilizadas como bordadura. As bandejas foram dispostas em armação de ferro, sendo instaladas no viveiro telado com sombrite de $50 \%$ de luminosidade.

O experimento foi conduzido em duas etapas, sendo realizado a avaliação inicial, que ocorreu em campo desde a semeadura, sendo avaliado Índice de Velocidade de Emergência (IVE) aos 15, 20, 25, 30 dias. A avaliação final sucedeu aos 41 DAS (dias após a semeadura), as plântulas foram retiradas das bandejas com 4-6 folhas definitivas, posteriormente analisou-se as características da plântula. A avaliação inicial das plântulas emergidas começou uma semana após a semeadura, sendo realizada diariamente a contagem considerando as plântulas totalmente emergidas somente as acima do substrato e com cotilédones abertos. A porcentagem de germinação foi calculada de acordo com Labouriau e Valadares (1976). Para o Índice de Velocidade de Emergência (IVE) e o Tempo Médio de Emergência (TME), determinados a partir da contagem diária, adotou-se a fórmula proposta em Maguire (1962).

A avaliação final ocorreu 41 DAS analisando-se as seguintes características: número de folhas (NF), altura da parte aérea (APA), diâmetro do colo (DC), massa fresca da parte aérea (MFPA), raiz (MFR) e total (MFT), massa seca da parte aérea (MSPA), raiz (MSR) e total (MST). Para obtenção das massas secas, o material vegetal foi colocado separadamente em estufa de circulação de ar forçado a $65{ }^{\circ} \mathrm{C}$ até atingir massa constante.

Para o bom desenvolvimento das plântulas a execução de diversos tratos culturais foram adotados para que não houvesse 0 comprometimento do vigor da muda. Para tanto, no presente experimento o controle de plantas invasoras e insetos foi realizado manualmente, e para a irrigação utilizou-se um regador, em que as mesmas foram irrigadas duas vezes ao dia. Aos 17 DAS foi efetuado o desbaste das plântulas, deixando em cada célula apenas a mais vigorosa.

Os dados foram submetidos ao teste de normalidade (Shapiro-Wilk) e homogeneidade (Bartlett) e quando significativos $(p>0,05)$ foi realizada análise de variância e as médias comparadas pelo teste de Tukey ao nível de 5\% de probabilidade. As análises foram realizadas pelo programa computacional Sistema para Análise de Variância - SISVAR ${ }^{\circledR}$ (FERREIRA, 2011).

\section{Resultados e Discussão \\ Produção de mudas de pimenta}

As cultivares utilizadas não apresentaram diferença para a porcentagem de emergência (E\%) e índice de velocidade de emergência (IVE). Em relação aos substratos houve diferença para a porcentagem de emergência em todo o período de avaliação, já o IVE apenas foi significativo para os dias 25 e 30, a nível de $5 \%$. A interação entre substrato e cultivar para a porcentagem de emergência não foi significativa aos 15 dias, no entanto aos 20, 25, 30 DAS foi significativa ao nível de $5 \%$.

Para o índice de velocidade de emergência nos 15 e 20 DAS, nenhum dos substratos diferiram entre si, porém aos 25 dias destacou-se o substrato comercial (testemunha), já aos 30 dias destacou-se o substrato comercial e $\mathrm{SC}+\mathrm{CAC}+\mathrm{HM}$ tiveram maior eficiência IVE, porém não diferindo entre si (Tabela 2). Resultados similares foram obtidos por Smiderle et al. (2001), onde destacam que o substrato comercial Plantmax apresentou melhor resultado para o IVE de mudas de pimentão. Substratos comerciais, em sua maioria, possuem características físicas e químicas favoráveis para o desenvolvimento inicial de plantas. Contudo, pode se tornar oneroso, principalmente quando se trata de pequenos produtores. 
Tabela 2. Índice de velocidade de emergência aos 25 (IVE 25) e 30 (IVE 30) dias e porcentagem de emergência aos 15 (E 15), 20 (E 20), 25 (E 25) e 30 (E 30) dias após a semeadura de sementes de cultivares de pimentas em diferentes substratos.

\begin{tabular}{|c|c|c|c|c|}
\hline Substratos & \multicolumn{2}{|c|}{ IVE 25} & \multicolumn{2}{|c|}{ IVE 30} \\
\hline SC & \multicolumn{2}{|c|}{$43,80 \mathrm{a}$} & \multicolumn{2}{|c|}{$52,33 \mathrm{a}$} \\
\hline$A+C A C+E B$ & \multicolumn{2}{|c|}{$28,66 \mathrm{c}$} & \multicolumn{2}{|c|}{$34,34 \mathrm{~b}$} \\
\hline $\mathrm{A}+\mathrm{CAC}+\mathrm{HM}$ & \multicolumn{2}{|c|}{$32,51 \mathrm{c}$} & \multicolumn{2}{|c|}{$39,31 \mathrm{~b}$} \\
\hline $\mathrm{SC}+\mathrm{CAC}+\mathrm{EB}$ & \multicolumn{2}{|c|}{$30,30 \mathrm{c}$} & \multicolumn{2}{|c|}{$36,21 \mathrm{~b}$} \\
\hline $\mathrm{SC}+\mathrm{CAC}+\mathrm{HM}$ & \multicolumn{2}{|c|}{$37,35 \mathrm{~b}$} & \multicolumn{2}{|c|}{$44,90 \mathrm{ab}$} \\
\hline CV (\%) & \multicolumn{2}{|c|}{34,99} & \multicolumn{2}{|c|}{30,96} \\
\hline \multirow{2}{*}{ Substratos } & \multicolumn{2}{|c|}{ E 15} & \multicolumn{2}{|c|}{ E 20} \\
\hline & PV & PA & PV & PA \\
\hline SC & $71,25 \mathrm{Aa}$ & $60,41 \mathrm{Aa}$ & $87,08 \mathrm{Aa}$ & 64,17 Bab \\
\hline$A+C A C+E B$ & $32,41 \mathrm{Ab}$ & 34,58 Aa & $41,25 \mathrm{Ab}$ & $37,66 \mathrm{Ab}$ \\
\hline $\mathrm{A}+\mathrm{CAC}+\mathrm{HM}$ & $52,50 \mathrm{Aab}$ & 48,33 Aa & $57,50 \mathrm{Aab}$ & 58,33 Aab \\
\hline $\mathrm{SC}+\mathrm{CAC}+\mathrm{EB}$ & $34,58 \mathrm{Ab}$ & $60,00 \mathrm{Aa}$ & $36,25 \mathrm{Bb}$ & 66,67 Aab \\
\hline $\mathrm{SC}+\mathrm{CAC}+\mathrm{HM}$ & $60,83 \mathrm{Aab}$ & 59,17 Aa & $65,00 \mathrm{Aab}$ & $71,25 \mathrm{Aa}$ \\
\hline CV (\%) & & & & \\
\hline \multirow{2}{*}{ Substratos } & \multicolumn{2}{|c|}{ E 25} & \multicolumn{2}{|c|}{ E 30} \\
\hline & PV & PA & PV & PA \\
\hline SC & $88,75 \mathrm{Aa}$ & $70,00 \mathrm{Aab}$ & 88,75 Aab & $70,00 \mathrm{Aa}$ \\
\hline$A+C A C+E B$ & $47,91 \mathrm{Ab}$ & $40,42 \mathrm{Ab}$ & 47,91 Ab & $40,42 \mathrm{Ab}$ \\
\hline $\mathrm{A}+\mathrm{CAC}+\mathrm{HM}$ & $60,41 \mathrm{Aab}$ & $66,25 \mathrm{Aab}$ & $60,41 \mathrm{Aab}$ & $66,25 \mathrm{Aab}$ \\
\hline $\mathrm{SC}+\mathrm{CAC}+\mathrm{EB}$ & $39,58 \mathrm{Bb}$ & $68,66 \mathrm{Aab}$ & $39,58 \mathrm{Bb}$ & $70,41 \mathrm{Aab}$ \\
\hline $\mathrm{SC}+\mathrm{CAC}+\mathrm{HM}$ & $65,00 \mathrm{Aab}$ & $75,42 \mathrm{Aa}$ & $65,00 \mathrm{Aab}$ & $75,42 \mathrm{Aa}$ \\
\hline CV (\%) & \multicolumn{2}{|c|}{24,10} & \multicolumn{2}{|c|}{24,10} \\
\hline
\end{tabular}

Médias seguidas de mesma letra minúscula na coluna e maiúscula na linha não diferem entre si pelo teste de Tukey a $5 \%$ de probabilidade. SC: Substrato comercial; A+CAC+EB: areia fina lavada + casca de arroz carbonizada + esterco bovino; $\mathrm{A}+\mathrm{CAC}+\mathrm{HM}$ : areia fina lavada + casca de arroz carbonizada + húmus de minhoca; SC+CAC+EB: substrato comercial + casca de arroz carbonizada + esterco bovino; SC+CAC+HM: substrato comercial + casca de arroz carbonizada + húmus de minhoca. Pimenta vermelha (PV) e amarela (PA).

Segundo Silva et al. (2011), o nível de hidratação adequado é fundamental para que se tenha uma boa germinação, pois nessa condição os processos metabólicos são ativados, o que resulta no desenvolvimento satisfatório do eixo embrionário. Sendo assim, substratos que possuem boa retenção de água podem favorecer a germinação e, consequentemente, o estabelecimento da plântula.

Em relação aos substratos alternativos testados, houve maior destaque para $\mathrm{O}$ $\mathrm{SC}+\mathrm{CAC}+\mathrm{HM}$ que se destacou no índice de velocidade de emergência (IVE). Possivelmente, essa combinação proporcionou melhores características físicas e químicas, semelhante ao substrato comercial (testemunha). De acordo com Silva et al. (2008), o substrato comercial proporciona a melhor formação de mudas de alta qualidade, o que garante o vigor e sanidade destas, além de reduzir o ciclo de produção.

Observou-se que a porcentagem de emergência (Tabela 2) do substrato comercial aos 15 DAS foi superior para as cultivares de pimenta amarela e vermelha, seguido do $\mathrm{SC}+\mathrm{CAC}+\mathrm{HM}$. Aos 20 DAS, para a cultivar de pimenta vermelha, o substrato comercial proporcionou a melhor emergência, seguido do $\mathrm{SC}+\mathrm{CAC}+\mathrm{EB}$. Para a cultivar de pimenta amarela em relação a 
testemunha verificou-se que o $\mathrm{SC}+\mathrm{CAC}+\mathrm{HM}$ foi o melhor, diferindo apenas do $\mathrm{A}+\mathrm{CAC}+\mathrm{EB}$. $\mathrm{O}$ húmus de minhoca é um composto rico em minerais que podem estar prontamente disponível para ser assimilados pelas plantas, visto que já passou por um processo de mineralização pelas minhocas. Rocha et al. (2007) encontraram resultados positivos com o uso de húmus de minhoca na produção de mudas de tomateiro, com germinação acima de $90 \%$.

Aos 25 e 30 DAS, observou-se que ocorreu a estabilização da porcentagem de emergência. A emergência da testemunha (substrato comercial Carolina Soil ${ }^{\circ}$ ) foi a melhor, sendo que entre os substratos alternativos testados, o que permaneceu mais próximo da testemunha foi substrato composto por $\mathrm{SC}+\mathrm{CAC}+\mathrm{HM}$. Sendo que a cultivar de pimenta vermelha teve a porcentagem de $65 \%$ de emergência aos 30 DAS, e para a pimenta amarela $75,42 \%$.
Verificou-se que a presença do húmus de minhoca nos compostos proporcionou uma melhor emergência. Segundo Medeiros et al. (2001), estudando os efeitos de substratos alternativos comparando ao comercial na produção de mudas de alface, verificou maior eficiência da mistura entre húmus de minhoca + casca de arroz carbonizada em relação aos demais compostos utilizados.

Em relação à altura da parte aérea, número de folhas e diâmetro do colo, as cultivares tiveram significância ao nível de $5 \%$. Todas as variáveis supracitadas apresentaram interação significativa (Tabela 3). Para a altura da parte aérea da plântula, os maiores valores foram obtidos à base do substrato comercial, a mistura composta por $\mathrm{SC}+\mathrm{P}+\mathrm{H}$ obteve a APA próximo ao valor da testemunha. A altura da planta é um dos aspectos mais importantes para medir o padrão de qualidade das mudas (SILVA et al., 2011).

Tabela 3. Altura da parte aérea (APA), número de folhas (NF), diâmetro do colo (DC), massa fresca da parte aérea (MFPA), raiz (MFR) e total (MFT), massa seca da parte aérea (MSPA), raiz (MSR) e total (MST) de plântula de pimenta em função de substratos e cultivares.

\begin{tabular}{ccccccc}
\hline \multirow{2}{*}{ Substratos } & \multicolumn{2}{c}{ APA (cm) } & \multicolumn{2}{c}{ NF } & \multicolumn{2}{c}{ DC (mm) } \\
\cline { 2 - 7 } & PV & PA & PV & PA & PV & PA \\
\hline SC & $3,33 \mathrm{Aa}$ & $1,69 \mathrm{Ba}$ & $3,78 \mathrm{Ba}$ & $4,81 \mathrm{Aa}$ & $0,66 \mathrm{Aa}$ & $0,56 \mathrm{Aa}$ \\
$\mathrm{A}+\mathrm{CAC}+\mathrm{EB}$ & $1,99 \mathrm{Ab}$ & $1,37 \mathrm{Aa}$ & $3,10 \mathrm{Ba}$ & $4,40 \mathrm{Aab}$ & $0,56 \mathrm{Aa}$ & $0,45 \mathrm{Aa}$ \\
$\mathrm{A}+\mathrm{CAC}+\mathrm{HM}$ & $2,35 \mathrm{Aab}$ & $1,10 \mathrm{Ba}$ & $2,90 \mathrm{Ba}$ & $3,79 \mathrm{Abc}$ & $0,59 \mathrm{Aa}$ & $0,48 \mathrm{Aa}$ \\
SC+CAC+EB & $2,74 \mathrm{Aab}$ & $1,13 \mathrm{Ba}$ & $3,02 \mathrm{Aa}$ & $3,49 \mathrm{Abc}$ & $0,71 \mathrm{Aa}$ & $0,45 \mathrm{Ba}$ \\
SC+CAC+HM & $2,48 \mathrm{Aab}$ & $1,39 \mathrm{Ba}$ & $2,85 \mathrm{Aa}$ & $3,29 \mathrm{Ac}$ & $0,62 \mathrm{Aa}$ & $0,48 \mathrm{Ba}$ \\
\hline CV (\%) & \multicolumn{2}{c}{24,54} & \multicolumn{2}{c}{13,60} & \multicolumn{2}{c}{14,50} \\
\hline Substratos & MFPA (g) & MFR(g) & MFT (g) & MSPA (g) & MSR (g) & MST (g) \\
\hline SC & $0,72 \mathrm{a}$ & $1,35 \mathrm{a}$ & $2,09 \mathrm{a}$ & $0,10 \mathrm{a}$ & $0,11 \mathrm{a}$ & $0,21 \mathrm{a}$ \\
$\mathrm{A}+\mathrm{P}+\mathrm{E}$ & $0,45 \mathrm{ab}$ & $0,62 \mathrm{ab}$ & $1,06 \mathrm{ab}$ & $0,03 \mathrm{~b}$ & $0,05 \mathrm{~b}$ & $0,08 \mathrm{~b}$ \\
A+P+H & $0,44 \mathrm{ab}$ & $0,54 \mathrm{~b}$ & $0,98 \mathrm{~b}$ & $0,05 \mathrm{ab}$ & $0,04 \mathrm{~b}$ & $0,90 \mathrm{ab}$ \\
SC+P+E & $0,58 \mathrm{ab}$ & $0,60 \mathrm{ab}$ & $1,18 \mathrm{ab}$ & $0,07 \mathrm{ab}$ & $0,05 \mathrm{~b}$ & $0,12 \mathrm{ab}$ \\
SC+P+H & $037 \mathrm{~b}$ & $0,51 \mathrm{~b}$ & $0,88 \mathrm{~b}$ & $0,05 \mathrm{ab}$ & $0,05 \mathrm{~b}$ & $0,10 \mathrm{ab}$ \\
\hline CV (\%) & 45,55 & 33,89 & 49,37 & 44,94 & 46,96 & 45,89 \\
\hline
\end{tabular}

Médias seguidas de mesma letra minúscula na coluna e maiúscula na linha não diferem entre si pelo teste de Tukey a 5\% de probabilidade. SC: Substrato comercial; A+CAC+EB: areia fina lavada + casca de arroz carbonizada + esterco bovino; A+CAC+HM: areia fina lavada + casca de arroz carbonizada + húmus de minhoca; SC+CAC+EB: substrato comercial + casca de arroz carbonizada + esterco bovino; SC+CAC+HM: substrato comercial + casca de arroz carbonizada + húmus de minhoca. Pimenta vermelha (PV) e amarela (PA).

O maior número de folhas (NF) foi observado nas mudas produzidas com a testemunha (substrato comercial), já para os substratos alternativos, o substrato composto por A+CAC+EB (Tabela 3). Possivelmente isso pode ter acontecido devido o balanço nutricional 
observado nos substratos supracitados. Santos et al. (2010) e Smiderle et al. (2001) também verificaram que o substrato comercial foi mais promissor para a número de folhas de mudas de pimentão, alface e pepino.

Em relação ao diâmetro do colo, o substrato $\mathrm{SC}+\mathrm{CAC}+\mathrm{EB}$ condicionou maior $\mathrm{DC}(0,71$ $\mathrm{mm}$ ) para a cultivar de pimenta vermelha, porém não diferiu dos outros substratos. A pimenta amarela obteve maior DC $(0,56 \mathrm{~mm})$ com substrato comercial (Tabela 3). Freitas et al. (2013), estudando a produção de mudas de alface em diferentes compostos alternativos verificaram que o crescimento do $D C$ foi superior em relação ao substrato Plantmax. De acordo com o mesmo estudo, o diâmetro do caule é um indicador da qualidade da muda para sobrevivência após o transplante. O diâmetro do colo é uma característica desejável das mudas, sendo um indicador de padrão de qualidade, pois confere maior sustentação (MARQUES et al., 2018) e estabelecimento das mudas ao serem transplantas.

Em relação a cultivar não houve diferença para as cultivares em relação a MFPA, MFR, MFT, MSPA, MSR, MST, já os substratos foram significativos. Verificou-se que não ocorreu interação entre os substratos e as cultivares.

Observou-se que o substrato comercial proporcionou maior produção de massa fresca da parte aérea $(0,72 \mathrm{~g})$, massa fresca da raiz $(1,35 \mathrm{~g})$, e massa fresca total $(2,09 \mathrm{~g})$, sendo superior aos demais substratos (Tabela 3). A mistura que propiciou o menor desenvolvimento de MFPA, MFR e MFT foi o substrato SC+CAC+HM. O mesmo ocorreu para MSPA, MSR e MST para o substrato comercial. Araújo Neto et al. (2009), estudando a produção de muda de pimentão em substrato diferentes substratos, observaram que o substrato comercial teve efeito superior em todas as características analisadas, uma vez que o substrato comercial apresenta composição química balanceada.

Dentre os substratos alternativos 0 substrato $S C+C A C+E B$ foi $O$ que apresentou melhor desempenho para a MFT e MST, efeito esse que pode estar relacionado a composição do substrato, pois a casca de arroz carbonizada proporcionou maior porosidade e aeração, consequentemente maior retenção de água. 0 esterco bovino agregado ao substrato comercial aumentou os teores de matéria orgânica e de nutrientes, assim complementaram os efeitos físico-hídricos da casca de arroz carbonizada, além disso a mistura apresentou bons teores de $\mathrm{P}$ e $\mathrm{K}$, contribuindo para um bom desenvolvimento das mudas.

\section{Produção de mudas de pimentão}

Os diferentes tipos de substratos analisados obtiveram uma diferença a nível de $1 \%$ de probabilidade e para as cultivares utilizadas observou-se diferença a nível de $5 \%$ de probabilidade para IVE aos 15 e 30 dias após a semeadura (DAS), e à nível de $1 \%$ para os IVE de 20 e 25 DAS. Houve diferença para a interação entre substratos e cultivares ao nível de $5 \%$. Resultados diferentes foram obtidos por Santos et al. (2010) e Silva et al. (2008) que, estudando a produção de mudas de pimentão, verificaram que não houve interação significativa entre o substrato e a cultivar. Para IVE o substrato comercial e a cultivar de pimentão amarelo apresentaram maior eficiência em todas as avaliações (Tabela 4). 
Tabela 4. Índice de velocidade de emergência aos 15 (IVE 15), 20 (IVE 20), 25 (IVE 25), e 30 (IVE 30) e emergência aos 15 (E 15), 20 (E 20), 25 (E 25) e 30 (E30) dias após a semeadura de cultivares de pimentão em função de substratos e cultivares.

\begin{tabular}{|c|c|c|c|c|c|c|}
\hline \multirow{2}{*}{ Substratos } & \multicolumn{3}{|c|}{ IVE 15} & \multicolumn{3}{|c|}{ IVE 20} \\
\hline & PV & PA & PVerde & PV & PA & PVerde \\
\hline SC & $25,98 \mathrm{Ba}$ & $40,13 \mathrm{Aa}$ & $25,64 \mathrm{Ba}$ & $35,00 \mathrm{Ba}$ & $54,35 \mathrm{Aa}$ & $34,49 \mathrm{Ba}$ \\
\hline$A+C A C+E B$ & $9,08 \mathrm{Ab}$ & $9,75 \mathrm{Ab}$ & $7,25 \mathrm{Ab}$ & $12,17 \mathrm{Ab}$ & $13,13 \mathrm{Ab}$ & $9,20 \mathrm{Ab}$ \\
\hline $\mathrm{A}+\mathrm{CAC}+\mathrm{HB}$ & $9,46 \mathrm{Ab}$ & $10,53 \mathrm{Ab}$ & $3,86 \mathrm{Ab}$ & $13,50 \mathrm{Ab}$ & $14,72 \mathrm{Ab}$ & $3,42 \mathrm{Ab}$ \\
\hline $\mathrm{SC}+\mathrm{CAC}+\mathrm{EB}$ & $11,99 \mathrm{Ab}$ & $7,48 \mathrm{Ab}$ & $6,83 \mathrm{Ab}$ & $13,77 \mathrm{Ab}$ & $9,31 \mathrm{Ab}$ & $8,42 \mathrm{Ab}$ \\
\hline $\mathrm{SC}+\mathrm{CAC}+\mathrm{HM}$ & $8,80 \mathrm{Ab}$ & $13,65 \mathrm{Ab}$ & $12,18 \mathrm{Ab}$ & $11,63 \mathrm{Ab}$ & $19,99 \mathrm{Ab}$ & $18,27 \mathrm{Ab}$ \\
\hline CV (\%) & & 40,42 & & & 41,03 & \\
\hline \multirow{2}{*}{ Substratos } & \multicolumn{3}{|c|}{ IVE 25} & \multicolumn{3}{|c|}{ IVE 30} \\
\hline & PV & PA & PVerde & PV & PA & PVerde \\
\hline SC & $44,42 \mathrm{Ba}$ & $67,44 \mathrm{Aa}$ & $44,47 \mathrm{Ba}$ & $52,48 \mathrm{Ba}$ & 78,19 Aa & $53,13 \mathrm{Ba}$ \\
\hline$A+C A C+E B$ & $17,12 \mathrm{Ab}$ & $18,76 \mathrm{Ab}$ & $13,70 \mathrm{Abc}$ & $21,86 \mathrm{Ab}$ & $24,21 \mathrm{Ab}$ & $18,27 \mathrm{Abc}$ \\
\hline $\mathrm{A}+\mathrm{CAC}+\mathrm{HM}$ & $19,64 \mathrm{Ab}$ & $20,86 \mathrm{Ab}$ & $5,52 \mathrm{Ac}$ & $25,14 \mathrm{ABb}$ & $26,43 \mathrm{Ab}$ & $7,68 \mathrm{Bc}$ \\
\hline $\mathrm{SC}+\mathrm{CAC}+\mathrm{EB}$ & $16,92 \mathrm{Ab}$ & $13,34 \mathrm{Ab}$ & $13,23 \mathrm{Abc}$ & $19,59 \mathrm{Ab}$ & $17,11 \mathrm{Ab}$ & $17,86 \mathrm{Abc}$ \\
\hline $\mathrm{SC}+\mathrm{CAC}+\mathrm{HM}$ & $16,41 \mathrm{Ab}$ & $27,43 \mathrm{Ab}$ & $26,33 \mathrm{Aab}$ & $20,88 \mathrm{Ab}$ & $33,84 \mathrm{Ab}$ & $20,88 \mathrm{Aab}$ \\
\hline CV (\%) & & 36,93 & & & 34,37 & \\
\hline \multirow{2}{*}{ Substratos } & \multicolumn{3}{|c|}{ E 15} & \multicolumn{3}{|c|}{ E 20} \\
\hline & PV & PA & PVerde & PV & PA & PVerde \\
\hline SC & $67,00 \mathrm{Ba}$ & $97,50 \mathrm{Aa}$ & $68,25 \mathrm{Ba}$ & $70,00 \mathrm{Ba}$ & $100,00 \mathrm{Aa}$ & $70,75 \mathrm{Bb}$ \\
\hline$A+C A C+E B$ & $31,75 \mathrm{Ab}$ & $33,00 \mathrm{Abc}$ & $26,25 \mathrm{Abc}$ & $33,75 \mathrm{Ab}$ & $35,50 \mathrm{Ab}$ & $27,75 \mathrm{Ab}$ \\
\hline $\mathrm{A}+\mathrm{CAC}+\mathrm{HM}$ & $34,25 \mathrm{Ab}$ & $37,75 \mathrm{Abc}$ & $8,75 \mathrm{Bc}$ & $40,50 \mathrm{Ab}$ & $41,50 \mathrm{Ab}$ & $13,50 \mathrm{Bb}$ \\
\hline $\mathrm{SC}+\mathrm{CAC}+\mathrm{EB}$ & $37,50 \mathrm{Ab}$ & $23,25 \mathrm{Ac}$ & $22,00 \mathrm{Abc}$ & $22,25 \mathrm{Ab}$ & $27,50 \mathrm{Ab}$ & $28,0 \mathrm{Ab}$ \\
\hline $\mathrm{SC}+\mathrm{CAC}+\mathrm{HM}$ & $33,00 \mathrm{Ab}$ & $52,25 \mathrm{Ab}$ & $42,75 \mathrm{Aab}$ & $33,50 \mathrm{Bb}$ & $53,75 \mathrm{ABb}$ & $58,75 \mathrm{Aa}$ \\
\hline CV (\%) & & 34,07 & & & 32,52 & \\
\hline \multirow{2}{*}{ Substratos } & \multicolumn{3}{|c|}{ E 25} & \multicolumn{3}{|c|}{ E 30} \\
\hline & PV & PA & PVerde & PV & PA & PVerde \\
\hline SC & $73,75 \mathrm{Ba}$ & $100,00 \mathrm{Aa}$ & $78,25 \mathrm{ABa}$ & $75,25 \mathrm{Ba}$ & $100,0 \mathrm{Aba}$ & $80,75 \mathrm{Aa}$ \\
\hline$A+C A C+E B$ & $39,00 \mathrm{Ab}$ & $47,50 \mathrm{Ab}$ & $39,50 \mathrm{Abc}$ & $44,25 \mathrm{Ab}$ & $51,0 \mathrm{Ab}$ & $43,0 \mathrm{Abc}$ \\
\hline $\mathrm{A}+\mathrm{CAC}+\mathrm{HM}$ & 49,75 Aab & $49,75 \mathrm{Ab}$ & $18,25 \mathrm{Bc}$ & $51,75 A a b$ & $52,50 \mathrm{Ab}$ & $20,50 \mathrm{Bc}$ \\
\hline $\mathrm{SC}+\mathrm{CAC}+\mathrm{EB}$ & $23,00 \mathrm{Ab}$ & $32,00 \mathrm{Ab}$ & $41,50 \mathrm{Abc}$ & $24,50 \mathrm{Bb}$ & $36,50 \mathrm{Ab}$ & $43,5 \mathrm{ABbc}$ \\
\hline $\mathrm{SC}+\mathrm{CAC}+\mathrm{HM}$ & $41,50 \mathrm{Bb}$ & $58,50 \mathrm{ABb}$ & $65,75 \mathrm{Aab}$ & $41,7 \mathrm{Ab}$ & $60,0 \mathrm{Ab}$ & $68,8 \mathrm{Aab}$ \\
\hline CV (\%) & \multicolumn{3}{|c|}{26,44} & \multicolumn{3}{|c|}{26,72} \\
\hline
\end{tabular}

Médias seguidas de mesma letra minúscula na coluna e maiúscula na linha não diferem entre si pelo teste de Tukey a $5 \%$ de probabilidade. SC: Substrato comercial; A+CAC+EB: areia fina lavada + casca de arroz carbonizada + esterco bovino; $\mathrm{A}+\mathrm{CAC}+\mathrm{HM}$ : areia fina lavada + casca de arroz carbonizada + húmus de minhoca; SC+CAC+EB: substrato comercial + casca de arroz carbonizada + esterco bovino; SC+CAC+HM: substrato comercial + casca de arroz carbonizada + húmus de minhoca. Pimentão vermelho (PV), amarelo (PA) e verde (Pverde).

Em relação aos substratos alternativos, a cultivar de pimentão verde teve alternância de resposta entre as avaliações do IVE, sendo que
IVE aos 15, 20 DAS foram melhores com o substrato $\mathrm{SC}+\mathrm{CAC}+\mathrm{EB}$, nas avaliações de IVE aos 25 e 30 DAS obteve melhor resposta com 
$\mathrm{A}+\mathrm{CAC}+\mathrm{HM}$. As cultivares de pimentão amarelo e vermelho responderam melhor à mistura de $\mathrm{SC}+\mathrm{CAC}+\mathrm{HM}$ em todas avalições de IVE.

As cultivares de pimentão amarelo e vermelho responderam melhor à mistura de $\mathrm{SC}+\mathrm{CAC}+\mathrm{HM}$ em todas avalições de IVE. Aos 15 DAS a porcentagem de emergência foi significativa a nível de $1 \%$, aos 20,25 e 30 DAS teve probabilidade de 5\%. Costa et al. (2013) destacam que a associação entre esterco bovino e substrato comercial apresentou melhor porcentagem de emergência de plantas de pimentão. A adição de fontes de matéria orgânica, como o esterco, contribui para o fornecimento de nutrientes e para a melhoria das características físicas do meio de cultivo, além de ser um insumo disponível aos produtores a baixo custo (SOUZA et al., 2015).

O substrato comercial teve maior eficiência e uniformidade na porcentagem de emergência para todas as cultivares e para a cultivar amarela a porcentagem de emergência foi de $100 \%$. Tal fato foi constatado por Rosa et al. (2011) ao avaliarem diferentes substratos comerciais para a produção de mudas de pimentão. Aos 15 DAS a cultivar de pimentão amarelo apresentou a melhor porcentagem de emergência $(97,50 \%)$, e aos 20 DAS todas as sementes emergiram e se manteve estabilizada até aos 25 e 30 DAS. A cultivar de pimentão vermelho não diferiu do pimentão amarelo aos 25, 30 DAS, já o pimentão verde apresentou a menor porcentagem de emergência em relação às demais cultivares (Tabela 4).

Batista et al. (2015), salientaram que a germinação rápida e homogênea são características fundamentais para a produção de mudas. Para porcentagem de emergência nos substratos alternativos, o composto por
$\mathrm{SC}+\mathrm{CAC}+\mathrm{HM}$ foi o melhor em todas as avaliações para as cultivares de pimentão amarelo e vermelho. A cultivar de pimentão verde aos 15 DAS se destacou o SC+CAC+EB. No entanto, para as avaliações aos 20,25 e 30 DAS o substrato $\mathrm{A}+\mathrm{CAC}+\mathrm{HM}$ apresentou maior porcentagem de emergência.

De acordo com Silva et al. (2008), os substratos compostos por areia, húmus de minhoca e substrato comercial condicionaram uma melhor porcentagem de germinação de plantas de alface. Gonçalves et al. (2016), ressalta que o teor de matéria orgânica nos resíduos animal, disponibiliza nutrientes beneficiando a germinação, consequentemente tendo uma plântula mais vigorosa.

Verificou-se que os substratos foram significativos para altura da parte aérea e número de folhas, já o diâmetro do colo não foi significativo. Não houve interação entre as cultivares e os substratos.

Conforme resultados encontrados na Tabela 5, verificou-se que o substrato comercial apresentou o melhor desenvolvimento para o número de folhas, o composto que apresentou menor número de folhas foi $\mathrm{SC}+\mathrm{CAC}+\mathrm{HM}$. Esse resultado está consoante Trani et al. (2004) ao avaliar a produção de mudas de alface em substratos comerciais. O balanço nutricional é de crucial importância para o crescimento inicial das mudas. Após o consumo das reservas oriundas da semente, a plântula necessita de um aporte nutricional para assegurar 0 seu bom desempenho. Além disso, o substrato deve acondicionar água de forma adequada, visto que a falta de água pode afetar os processos fisiológicos, bioquímicos e de expansão foliar. 
Tabela 5. Altura da parte aérea (APA), número de folhas (NF), diâmetro do colo (DC), massa fresca da parte aérea (MFPA), raiz (MFR) e total (MFT), massa seca da parte aérea (MSPA), raiz (MSR) e total (MST) de plântulas de pimentão em função dos diferentes substratos.

\begin{tabular}{|c|c|c|c|c|c|c|}
\hline Substratos & \multicolumn{2}{|c|}{ APA } & \multicolumn{2}{|c|}{ NF } & \multicolumn{2}{|c|}{ DC } \\
\hline SC & \multicolumn{2}{|c|}{$3,17 \mathrm{a}$} & \multicolumn{2}{|c|}{$4,16 \mathrm{a}$} & \multicolumn{2}{|c|}{$0,99 a$} \\
\hline $\mathrm{A}+\mathrm{CAC}+\mathrm{EB}$ & \multicolumn{2}{|c|}{$2,37 \mathrm{~b}$} & \multicolumn{2}{|c|}{$3,62 a b$} & \multicolumn{2}{|c|}{$1,05 \mathrm{a}$} \\
\hline $\mathrm{A}+\mathrm{CAC}+\mathrm{HM}$ & \multicolumn{2}{|c|}{$2,03 \mathrm{~b}$} & \multicolumn{2}{|c|}{$3,58 a b$} & \multicolumn{2}{|c|}{$0,97 a$} \\
\hline $\mathrm{SC}+\mathrm{CAC}+\mathrm{EB}$ & \multicolumn{2}{|c|}{$3,22 \mathrm{a}$} & \multicolumn{2}{|c|}{$3,97 \mathrm{a}$} & \multicolumn{2}{|c|}{$1,10 \mathrm{a}$} \\
\hline $\mathrm{SC}+\mathrm{CAC}+\mathrm{HM}$ & \multicolumn{2}{|c|}{$2,29 \mathrm{~b}$} & \multicolumn{2}{|c|}{$3,22 b$} & \multicolumn{2}{|c|}{$1,10 \mathrm{a}$} \\
\hline CV (\%) & \multicolumn{2}{|c|}{23,75} & \multicolumn{2}{|c|}{14,77} & \multicolumn{2}{|c|}{17,93} \\
\hline Substratos & MVPA & MVR & MVT & MSPA & MSR & MST \\
\hline SC & $1,54 a$ & $1,93 \mathrm{a}$ & $3,47 a$ & $0,81 \mathrm{a}$ & $0,73 a$ & $1,54 \mathrm{a}$ \\
\hline$A+C A C+E B$ & $0,67 b$ & $0,83 \mathrm{~b}$ & $1,50 \mathrm{~b}$ & $0,39 \mathrm{~b}$ & $0,34 \mathrm{~b}$ & $0,73 \mathrm{~b}$ \\
\hline $\mathrm{A}+\mathrm{CAC}+\mathrm{HM}$ & $0,52 \mathrm{~b}$ & $0,69 \mathrm{~b}$ & $1,21 b$ & $0,29 \mathrm{~b}$ & $0,24 \mathrm{~b}$ & $0,53 \mathrm{~b}$ \\
\hline $\mathrm{SC}+\mathrm{CAC}+\mathrm{EB}$ & $0,70 \mathrm{~b}$ & $0,96 \mathrm{~b}$ & $1,66 b$ & $0,42 b$ & $0,48 \mathrm{~b}$ & $0,90 \mathrm{~b}$ \\
\hline $\mathrm{SC}+\mathrm{CAC}+\mathrm{HM}$ & $0,61 b$ & $0,79 b$ & $1,40 \mathrm{~b}$ & $0,36 \mathrm{~b}$ & $0,45 b$ & $0,81 b$ \\
\hline CV (\%) & 46,18 & 51,63 & 50,29 & 39,6 & 49,43 & 40,96 \\
\hline
\end{tabular}

Médias seguidas de mesma letra minúscula na coluna não diferem entre si pelo teste de Tukey a $5 \%$ de probabilidade. SC: Substrato comercial; A+CAC+EB: areia fina lavada + casca de arroz carbonizada + esterco bovino; A+CAC+HM: areia fina lavada + casca de arroz carbonizada + húmus de minhoca; SC+CAC+EB: substrato comercial + casca de arroz carbonizada + esterco bovino; SC+CAC+HM: substrato comercial + casca de arroz carbonizada + húmus de minhoca.

O substrato comercial produziu diâmetro do colo inferior comparado com $\mathrm{SC}+\mathrm{CAC}+\mathrm{EB}, \mathrm{SC}+$ $\mathrm{CAC}+\mathrm{HM}$, mas não diferiram entre si. Santos et al. (2010), trabalharam com substratos alternativos para mudas de pimentão obtiveram resultados semelhantes, onde 0 substrato comercial proporcionou um diâmetro do colo inferior $(1,77 \mathrm{~mm})$ ao encontrado no vermicomposto.

Dentre os substratos alternativos foi observado que a maior altura da parte aérea e número de folhas foi propiciado pelo $\mathrm{SC}+\mathrm{CAC}+\mathrm{EB}$. Steffen et al. (2010), utilizaram casca de arroz e esterco bovino para a produção de mudas de alface e tomate, verificaram que as mudas cultivadas com maiores concentrações de casca de arroz carbonizada e esterco bovino tiveram o maior desenvolvimento na altura e o número de folhas. Segundo os mesmos autores, o teor de matéria orgânica assegura um elevado número de espaços poroso, proporcionando aeração e drenagem adequadas.

Não houve diferença para as cultivares quanto para interação cultivar e substratos para as variáveis de massas frescas e secas. Conforme a Tabela 5, o substrato comercial apresentou a maior produção para as variáveis avaliadas, diferindo estaticamente dos demais substratos estudados. $O$ substrato que teve a menor produção foi a mistura de $\mathrm{A}+\mathrm{CAC}+\mathrm{HM}$. Smiderle et al. (2001), constataram que o substrato comercial Plantmax foi o que promoveu maior produção de massa seca para todas as cultivares avaliadas.

O substrato alternativo que mais se aproximou do comercial foi o composto por $\mathrm{SC}+\mathrm{CAC}+\mathrm{EB}$, o resultado desse composto pode estar relacionado a disponibilidade de macronutrientes ser maior que as demais misturas, além de apresentar bons teores de matéria orgânica. $O$ acúmulo de biomassa seca está relacionado ao bom desempenho da plântulas em absorver nutrientes e de produzir fotoassamilados. Para um bom desempenho fotossintético, na fase inicial do crescimento vegetativo, é necessário que as plântulas estejam em condições ótimas de nutrição, luminosidade, disponibilidade hídrica, dentre outros. Assim, poderá particionar seus assimilados e transformar em matéria seca radicular e de parte aérea de modo a ter bom desempenho quando transplantada para local definitivo. 


\section{Conclusão}

O substrato comercial Carolina Soil ${ }^{\circ}$ foi o mais adequado para a produção de mudas de pimenta e pimentão. $O$ substrato alternativo composto por o substrato comercial + casca de arroz carbonizada + esterco bovino ( $\mathrm{SC}+\mathrm{CAC}+\mathrm{EB}$ ) pode ser utilizado quando se trata de uso de substratos alternativos para a produção de mudas das espécies supracitadas.

\section{Referências}

ALVES, H. Q.; ROSSETE, A. N.; GROSS, M. S. Caracterização do uso e cobertura da terra na microbacia hidrográfica do Córrego Murtinho, Nova Xavantina - MT. Caminhos de Geografia, v.12, n.8, p. 41-56, 2011.

ARAÚJO NETO, S. E.; AZEVEDO, J. M. A.; GALVÃO, R. O.; OLIVEIRA, E. B. L.; FERREIRA, R. L. F. Produção de muda orgânica de pimentão com diferentes substratos. Ciência Rural, v.39, n.5, p. 1408-1413, 2009.

https://doi.org/10.1590/S0103-

$\underline{84782009005000099}$

ARAÚJO, A. C.; ARAÚJO, A. C.; DANTAS, M. K. L.; PEREIRA, W. E.; ALOUFA, M. A. I. Utilização de substratos orgânicos na produção de mudas de mamoeiro formosa. Revista Brasileira de Agroecologia, v.8, n.1, p. 210-216, 2013.

BATISTA, T. B.; BINOTTI, F. F. S.; CARDOSO, E. D.; BARDIVIESSO, E. M.; COSTA, E. Aspectos fisiológicos e qualidade de mudas da pimenteira em resposta ao vigor e condicionamento das sementes. Bragantia, v.74, n.4, p. 367-373, 2015. https://doi.org/10.1590/1678-4499.0133

BÜTTOW, M. V.; BARBIERI, R. L.; NEITZKE, R. S.; HEIDEN, G.; CARVALHO, F. I. F. D. Diversidade genética entre acessos de pimentas e pimentões da Embrapa Clima Temperado. Ciência Rural, v.40, n.6, p. 1264-1269, 2010.

https://doi.org/10.1590/S0103-

84782010000600004

COSTA, E.; JORGE, M. H.; SCHWERZ, F.; CORTELASSI, J. A. D. S. Emergência e fitomassa de mudas de pimentão em diferentes substratos. Revista Brasileira de Ciências Agrárias, v.8, n.3, p. 396-401, 2013. https://doi.org/10.5039/agraria.v8i3a2428
COSTA, L. A. D. M.; COSTA, M. S. S. M.; PEREIRA, D. C.; BERNARDI, F. H.; MACCARI, S. Avaliação de substratos para a produção de mudas de tomate e pepino. Revista Ceres, v.60, n.5, p. 675-682, 2015. https://doi.org/10.1590/50034737X2013000500011

DINIZ, K.; GUIMARÃES, S. T. M. R.; LUZ, J. M. Q. Húmus como substrato para a produção de mudas de tomate, pimentão e alface. Bioscience Journal, v.22, n.3, p. 63-70, 2006.

FERREIRA, D. F. Sisvar: a computer statistical analysis system. Ciência e Agrotecnologia, v.35, n.6, p. 1039-1042, 2011. https://doi.org/10.1590/S1413$\underline{70542011000600001}$

FREITAS, G. A.; SILVA, R. R.; BARROS, H. B; MELO, A. V.; ABRAHÃO, W. A. P. Produção de mudas de alface em função de diferentes combinações de substratos. Revista Ciência Agronômica,

v.44, n.1, p. 159-166, 2013.

https://doi.org/10.1590/S1806-

$\underline{66902013000100020}$

GONÇALVES, F. C. M.; ARRUDA, F. P.; SOUSA, F. L.; ARAÚJO, J. R. Germinação e desenvolvimento de mudas de pimentão Cubanelle em diferentes substratos. Revista Mirante (UFG), v.9, n.1, p. 3545, 2016.

LABOURIAU, L. G.; VALADARES, M. E. B. On the germination of seeds Calotropis procera (Ait.) Ait.f. Anais da Academia Brasileira de Ciências, v.48, n.2, p. 263-284, 1976.

MACIEL, T. C. M.; SILVA, T. I.; ALCANTARA, F. D. O.; MARCO, C. A.; NESS, R. L. L. Substrato à base de pequi (Caryocar coriaceum) na produção de mudas de tomate e pimentão. Journal of Neotropical Agriculture, v.4, n.2, p. 9-16, 2017. https://doi.org/10.32404/rean.v4i2.1551

MAGUIRE, J. D. Speed of gerrnination - aid in selection and evaluation for seedling emergence and vigor. Crop Science, v.2, n.2, p. 176-177, 1962.

https://doi.org/10.2135/cropsci1962.0011183X0 $\underline{00200020033 \mathrm{x}}$

MARQUES, A. R. F.; DELOSS, A. M.; OLIVEIRA, V. S.; BOLIGON, A. A.; VESTENA, S. Produção e qualidade de mudas de Eugenia uniflora L. em 
diferentes substratos. Ambiência, v.14, n.1, p. 4456, 2018.

MEDEIROS, L. A. M.; MANFRON, P. A.; MEDEIROS, S. L. P.; BONNECARRÈRE, R. A. G. Crescimento e desenvolvimento da alface (Lactuca sativa L.) conduzida em estufa plástica com fertirrigação em substratos. Ciência Rural, v.31, n.2, p. 199204, 2001. https://doi.org/10.1590/S010384782001000200002

NADAI, F. B.; MENEZES, J. B. C.; CATÃO, H. C. R. M.; ADVÍnCULA, T.; COSTA, C. A. Produção de mudas de tomateiro em função de diferentes formas de propagação e substratos. Revista Agro@mbiente On-line, v.9, n.3, p. 261-267, $2015 . \quad$ https://doi.org/10.18227/19828470ragro.v9i3.2348

ROCHA, M. Q.; COGO, C. M.; OLANDA, R.B. Casca de arroz in natura como substrato para produção de mudas de tomateiro. Revista Brasileira de Agroecologia, v.2, n.2, p. 1208-1211, 2007.

ROSA, J. Q. S.; FERREIRA, R. C.; BALBINO, M. P. A.; TAVARES, T. R.; BEZERRA, R. S.; FARIAS, J. G. Produção de mudas de pimentão: substratos comerciais e fertirrigação. 2011. Dissertação (Mestrado em Agronomia) - Universidade Federal de Goiás, Goiânia, 2011. Disponível em: http://www.sbpcnet.org.br/livro/63ra/conpeex/ mestrado/trabalhos-mestrado/mestrado-julianoqueiroz.pdf. Acesso em: 04 mar. 2019.

SAIDELLES, F. L. F.; CALDEIRA, M. V. W.; SCHIRMER, W. N.; SPERANDIO, H. V. Casca de arroz carbonizada como substrato para produção de mudas de tamboril-da-mata e garapeira. Semina: Ciências Agrárias, v.30, n.1, p. 1173-1186, 2009. https://doi.org/10.5433/16790359.2009v30n4Sup1p1173

SANTOS, M. R.; SEDIYAMA, M. A. N.; SALGADO, L. T.; VIDIGAL, S. M.; REIGADO, F. R. Produção de mudas de pimentão em substratos à base de vermicomposto. Bioscience Journal, v.26, n.4, p. 572-578, 2010.

SILVA, E. A.; MENDONCA, V.; TOSTA, M. S.; OLIVEIRA, A. C.; REIS, L. L.; BARDIVIESSO, D. M. Germinação da semente e produção de mudas de cultivares de alface em diferentes substratos. Semina: Ciências Agrarias, v.29, n.2, p. 245-254,
2008. https://doi.org/10.5433/1679$\underline{0359.2008 v 29 n 2 p 245}$

SILVA, J. D. C.; LEAL, T. T. B.; ARAÚJO, R. M.; GOMES, R. L. F.; ARAÚJO, A. S. F.; MELO, W. J. Emergência e crescimento inicial de plântulas de pimenta ornamental e celosia em substrato à base de composto de lodo de curtume. Ciência Rural, v.41, n.3, p. 412-417, 2011.

https://doi.org/10.1590/S0103-

84782011000300008

SMIDERLE, O. J.; SALIBE, A. B.; HAYASHI, A. H.; MINAMI, K. Produção de mudas de alface, pepino e pimentão em substratos combinando areia, solo e Plantmax ${ }^{\circledR}$. Horticultura Brasileira, v.19, n.3, p. 253-257, 2001.

https://doi.org/10.1590/S0102-

$\underline{05362001000300022}$

STEFFEN, G. P. K.; ANTONIOLLI, Z. I.; STEFFEN, R. B.; MACHADO, R. G. Casca de arroz e esterco bovino como substratos para a multiplicação de minhocas e produção de mudas de tomate e alface. Acta Zoológica Mexicana, v.26, n.2, p. 333-343, 2010.

https://doi.org/10.21829/azm.2010.262898

SOUZA, E. G. F.; JÚNIOR, A. P. B.; SILVEIRA, L. M.; SANTOS, M. G.; SILVA, E. F. Emergência e desenvolvimento de mudas de tomate IPA 6 em substratos, contendo esterco ovino. Ceres, v.60, n.6, p. 902-907, 2015.

https://doi.org/10.1590/S0034-

737X2013000600020

TRANI, P. E.; NOVO, M. C. S. S.; CAVALLARO JÚNIOR, M. L.; TELLES, L. M. G. Produção de mudas de alface em bandejas e substratos comerciais. Horticultura Brasileira, v.22, n.2, p. 290-294, 2004. https://doi.org/10.1590/S010205362004000200025

TRENTO, E. J; SEPULCRI, O.; MORIMOTO, F. Comercialização de frutas, legumes e verduras. Curitiba: Instituto Emater, 2011. 40 p. (Série Informação Técnica, 85). Disponível em: http://www.asbraer.org.br/arquivos/bibl/79com.pdf. Acesso em: 04 mar. 2019. 